\title{
On Global Inversion Theorems in the Plane
}

\author{
Hong Ding \\ Faculty of Science, Beijing University of Technology, Beijing, PR China \\ E-mail: 13681492772@163.com
}

Received: 19 June 2021; Revised: 31 August 2021; Accepted: 01 September 2021

Abstract: Let $f=\left(f_{1}, f_{2}\right) \in C^{\infty}\left(\mathbb{R}^{2}, \mathbb{R}^{2}\right)$, we say that $f$ increases small distances if there exists $\varepsilon>0$ such that $\|a-b\|<\varepsilon$ implies $\|f(a)-f(b)\| \geq\|a-b\|, \forall a, b \in \mathbb{R}^{2}$. In this paper, we prove the following: Suppose $f$ increases small distances, then $f: \mathbb{R}^{2} \rightarrow \mathbb{R}^{2}$ is an onto diffeomorphism. We also get an equivalent version of the Hadamard's global inverse function theorem from our Theorem 4 .

Keywords: diffeomorphism, global inversion, maps that increase small distances

AMS: 57R50, 58C25

\section{Introduction}

In this paper, we are concerned with the question of when a $C^{\infty}$ map $f: \mathbb{R}^{2} \rightarrow \mathbb{R}^{2}$ becomes an onto diffeomorphism. A good introduction to this type of the topic can be found in [1].

We first improve some of our results in [2]. We actually use the same proofs, because the old proofs also work for our new and more generalized conditions. We then use this new result (Theorem 1) to work out some of the results in section 2 of this paper. Then in section 3, we use the theorems and propositions in section 2 to prove the theorems in section 3, which includes our main results.

In section 3, we first prove Theorem 4, which is kind of technical and useful. Actually, our Theorem 4 states the following:

Theorem 4 Let $f: \mathbb{R}^{2} \rightarrow \mathbb{R}^{2}, f \in C^{\infty}\left(\mathbb{R}^{2}, \mathbb{R}^{2}\right)$. Let $\operatorname{det}(D f(x))>0, \forall x \in \mathbb{R}^{2}$. Suppose there exists a positive number $d>0$, so that for every $x_{0} \in \mathbb{R}^{2}$, we have $a_{x_{0}} \in \mathbb{R}^{2}$ and $r_{x_{0}}>0$, so that $x_{0} \in B\left(a_{x_{0}}, r_{x_{0}}\right)$ and $f\left(\partial B\left(a_{x_{0}}, r_{x_{0}}\right)\right) \subset \mathbb{R}^{2} \backslash B\left(f\left(x_{0}\right), d\right)$. Then $f: \mathbb{R}^{2} \rightarrow \mathbb{R}^{2}$ is an onto diffeomorphism.

Here, $\operatorname{det}(D f(x))$ is the Jacobian of $f$. And $B(a, r)=\left\{x \in \mathbb{R}^{2}:\|x-a\|<r\right\}$.

As an application of Theorem 4, we prove one of our main results, Corollary 1, which is a version of Hadamard's global inverse function theorem in the plane. Actually, our Corollary 1 states the following:

Corollary 1 (A version of Hadamard's global inverse function theorem in the plane.) Let $f: \mathbb{R}^{2} \rightarrow \mathbb{R}^{2}, f \in C^{\infty}\left(\mathbb{R}^{2}\right.$, $\mathbb{R}^{2}$ ). Let $\operatorname{det}(D f(x))>0, \forall x \in \mathbb{R}^{2}$. Suppose $\lim _{\|x\| \rightarrow \infty}\|f(x)\|=\infty$. Then $f: \mathbb{R}^{2} \rightarrow \mathbb{R}^{2}$ is an onto diffeomorphism.

Then we get another main result: Theorem 5 which is an application of Theorem 4. Actually, our Theorem 5 states

Copyright (C2021 Hong Ding

DOI: https://doi.org/10.37256/cm.242021998

This is an open-access article distributed under a CC BY license

(Creative Commons Attribution 4.0 International License)

https://creativecommons.org/licenses/by/4.0/ 
the following:

Theorem 5 Let $f=\left(f_{1}, f_{2}\right) \in C^{\infty}\left(\mathbb{R}^{2}, \mathbb{R}^{2}\right)$. Suppose $f$ increases small distances, then $f: \mathbb{R}^{2} \rightarrow \mathbb{R}^{2}$ is an onto diffeomorphism.

The concept of a map that increases small distances is defined in our Definition 1. We give an example of the application of Theorem 5. This example is also a non-trivial example of a map that increases small distances.

\section{Preliminaries}

First, we need a few lemmas before we get to the theorems.

Lemma 1 Let $f=\left(f_{1}, f_{2}\right) \in C^{1}\left(\mathbb{R}^{2}, \mathbb{R}^{2}\right), i \in\{1,2\}, K \subset \mathbb{R}^{2}$ be a compact set and the Jacobian $\operatorname{det}(D f(x))>0, x \in K$. Then for every $c \in f_{i}(K)$, the 1-dimensional Hausdorff measure of $f_{i}^{-1}(c) \cap K$ is finite.

Proof. This is exactly Lemma 1 in [2], see the proof of Lemma 1 in [2].

Lemma 2 Let $f=\left(f_{1}, f_{2}\right): \mathbb{R}^{2} \rightarrow \mathbb{R}^{2}, f \in C^{\infty}\left(\mathbb{R}^{2} \rightarrow \mathbb{R}^{2}\right)$. Let $E \subset \mathbb{R}^{2}$ be a bounded region. And suppose $\operatorname{det}(D f(x))$ $>0, \forall x \in \bar{E}$. Suppose there exists $K>0, r \in(0, K]$ so that $f(\partial E) \subset \overline{B(0, K)} \backslash B(0, r)$ and $f^{-1}(B(0, r)) \cap E \neq \varnothing$. Let $\xi \in f$ ${ }^{-1}(B(0, r)) \cap E, c=f_{2}(\xi)$. Let $\Gamma$ be the connected component of $f_{2}^{-1}(c) \cap E$ containing $\xi$. Then $f(\Gamma) \supset\left\{(x, c) \in \mathbb{R}^{2}: x^{2}+\right.$ $\left.c^{2}<r^{2}\right\}$. Similar result exists for $f_{1}$.

Proof. The proof of Lemma 2 in [2] also works for Lemma 2 here. Because in the proof of Lemma 2 in [2] we just use the facts that $\overline{B(0, R)}$ is a compact set in $\mathbb{R}^{2}$ and $B(0, R)$ is open. Please read the proof of Lemma 2 in [2].

Lemma 3 Let $f=\left(f_{1}, f_{2}\right): \mathbb{R}^{2} \rightarrow \mathbb{R}^{2}, f \in C^{\infty}\left(\mathbb{R}^{2} \rightarrow \mathbb{R}^{2}\right)$. Let $E \subset \mathbb{R}^{2}$ be a bounded region. And suppose $\operatorname{det}(D f(x))>0$, $\forall x \in \bar{E}$. Suppose there exists $K>0, r \in(0, K]$ so that $f(\partial E) \subset B(0, K) \backslash B(0, r)$. Let $\lambda \in f(E) \cap B(0, r) \neq \varnothing, \mu \in f^{-1}(\lambda) \cap E$. Then there exists a continuous function $L: B(0, r) \rightarrow E$, so that $f(L(u))=u, \forall u \in B(0, r)$ and $L(\lambda)=\mu$.

Proof. Read the proof of Lemma 3 in [2]. When you see $B(0, R)$, substitute it by $E$. Substitute Lemma 2 in [2] by Lemma 2 here. Then you get a proof of Lemma 3 here.

Now we have the following proposition which is a slight generalization of Proposition 1 in [2].

Proposition 1 Let $f=\left(f_{1}, f_{2}\right) \in C^{\infty}\left(\mathbb{R}^{2}, \mathbb{R}^{2}\right)$. Let $E \subset \mathbb{R}^{2}$ be a bounded region. And suppose $\operatorname{det}(D f(x))>0, \forall x \in \bar{E}$. Suppose there exists $K>0, r \in(0, K]$ satisfying $f^{-1}(B(0, r)) \cap E \neq \varnothing$ and $f(\partial E) \subset \overline{B(0, K)} \backslash B(0, r)$. Let $H$ be any path connected component of $f^{-1}(B(0, r)) \cap E$. Then $\left.f\right|_{H}: H \rightarrow B(0, r)$ is an onto $C^{\infty}$-diffeomorphism.

Proof. Read the proof of Proposition 1 in [2], when you see $B(0, R)$, substitute it by $E$. When you see Lemma 3 of [2], substitute it by Lemma 3 here. Then you get a proof of Proposition 1 here.

The following theorem is a slight generalization of Theorem 1 in [2].

Theorem 1 Let $F=\left(F_{1}, F_{2}\right) \in C^{\infty}\left(\mathbb{R}^{2}, \mathbb{R}^{2}\right)$. Let $E \subset \mathbb{R}^{2}$ be a bounded region. And suppose the Jacobian $\operatorname{det}(D F(x))>0$, $\forall x \in \bar{E}$. Suppose there exists $K>0, r \in(0, K]$ and $a \in \mathbb{R}^{2}$ satisfying $F^{-1}(B(a, r)) \cap E \neq \varnothing$ and $F(\partial E) \subset \overline{B(a, K)} \backslash B(a, r)$. Let $H$ be any path connected component of $F^{-1}(B(a, r)) \cap E$. Then $\left.F\right|_{H}: H \rightarrow B(a, r)$ is an onto $C^{\infty}$-diffeomorphism.

Proof. Let $f(x)=F(x)-a, \forall x \in \mathbb{R}^{2}$. We can apply Proposition 1 to $f$. Then we get the conclusion.

Theorem 2 Let $f: \mathbb{R}^{2} \rightarrow \mathbb{R}^{2}, f \in C^{\infty}\left(\mathbb{R}^{2}, \mathbb{R}^{2}\right)$. Suppose $\operatorname{det}(D f(x))>0, \forall x \in \mathbb{R}^{2}$. Let $D=B(a, r), a \in \mathbb{R}^{2}, r>0$. Let $E$ be a bounded connected component of $f^{-1}(D)$. Then $\left.f\right|_{E}: E \rightarrow D$ is an onto diffeomorphism.

Proof. Because $f$ is continuous, $f^{-1}(D)$ is open in $\mathbb{R}^{2}$. Because $\mathbb{R}^{2}$ is locally connected, every connected component of the open set $f^{-1}(D)$ is open. So $E \subset f^{-1}(D)$ is open in $\mathbb{R}^{2}$. Therefore $E \subset \mathbb{R}^{2}$ is a bounded region.

Now let $\delta \in \partial E$. Let $\widetilde{E}$ be another connected component of $f^{-1}(D)$. Then $\delta \bar{\epsilon} \widetilde{E}$. Actually if $\delta \in \widetilde{E}$, then $\exists$ a neighborhood $U$ of $\delta$ such that $U \subset \widetilde{E}$ (for $\widetilde{E}$ is open). Because $\delta \in \partial E$, we have that $U \cap E \neq \varnothing$. Let $q \in U \cap E$, then $q \in \widetilde{E} \cap E$. This contradicts the fact that $\widetilde{E}$ and $E$ are different connected components of $f^{-1}(D)$. Therefore we have proven that $\delta \bar{\in} \widetilde{E}$. Certainly $\delta \bar{\in} E$. (For $E \subset \mathbb{R}^{2}$ is open.) Therefore $\delta$ is not in any of the connected components of $f^{-1}(D)$. So $f(\delta) \bar{\in} D$. 
Now we can see $\operatorname{det}(D f(x))>0, \forall x \in \bar{E}$. And $\exists K>r(>0)$, so that $f(\partial E) \subset \overline{B(a, K)} \backslash D$. Also $f^{-1}(D) \cap E=E \neq \varnothing$. Let $H$ be any path connected component of $f^{-1}(D) \cap E$. Then by Theorem $1,\left.f\right|_{H}: H \rightarrow D$ is an onto diffeomorphism.

Next we want to say that $E$ is a path connected component of $f^{-1}(D) \cap E$. Because all connected open sets of $\mathbb{R}^{n}$ are path connected, we see that $E$ is path connected. So $f^{-1}(D) \cap E=E$ is path connected. So the only path connected component of $f^{-1}(D) \cap E$ is $f^{-1}(D) \cap E$ itself. So $E$ is a path connected component of $f^{-1}(D) \cap E$. Therefore we have $\left.f\right|_{E}: E \rightarrow D$ is an onto diffeomorphism.

Next we have a proposition.

Proposition 2 Let $f: \mathbb{R}^{2} \rightarrow \mathbb{R}^{2}, f \in C^{\infty}\left(\mathbb{R}^{2}, \mathbb{R}^{2}\right)$. Let $\operatorname{det}(D f(x))>0, \forall x \in \mathbb{R}^{2}$. Suppose there exists $d>0$, so that for every $x_{0} \in \mathbb{R}^{2}$, there exist $a_{x_{0}} \in \mathbb{R}^{2}$ and $r_{x_{0}}>0$ so that $x_{0} \in B\left(a_{x_{0}}, r_{x_{0}}\right)$ and $f\left(\partial B\left(a_{x_{0}}, r_{x_{0}}\right)\right) \subset \mathbb{R}^{2} \backslash B\left(f\left(x_{0}\right), d\right)$. Then we get, $f: \mathbb{R}^{2} \rightarrow \mathbb{R}^{2}$ is an onto map.

Proof. Fix $f(0) \in \mathbb{R}^{2}$. Let $l$ be any half-line in $\mathbb{R}^{2}$ starting from $f(0)$. We want to show that $l \subset f\left(\mathbb{R}^{2}\right)$.

By our condition, $\exists a_{0} \in \mathbb{R}^{2}$ and $r_{0}>0$, so that $0 \in B\left(a_{0}, r_{0}\right)$ and $f\left(\partial B\left(a_{0}, r_{0}\right)\right) \subset \mathbb{R}^{2} \backslash B(f(0), d)$. Certainly, there exists $K>d$ such that $f\left(\partial B\left(a_{0}, r_{0}\right)\right) \subset \overline{B(f(0), K)} \backslash B(f(0), d)$. Let $H$ be a path connected component of $f^{-1}(B(f(0), d))$ $\cap B\left(a_{0}, r_{0}\right)$. By Theorem $1,\left.f\right|_{H}: H \rightarrow B(f(0), d)$ is an onto diffeomorphism. So $B(f(0), d) \subset f\left(\mathbb{R}^{2}\right)$.

Let $y_{1}$ be the point on $l$ for which $\left\|y_{1}-f(0)\right\|=\frac{d}{2}$. Then we can see that $y_{1}=f\left(\alpha_{1}\right)$ for some $\alpha_{1} \in \mathbb{R}^{2}$. By the same method above, we can prove that $B\left(y_{1}, d\right) \subset f\left(\mathbb{R}^{2}\right)$. Now let $y_{n}$ be the point on $l$ for which $\left\|y_{n}-f(0)\right\|=\frac{n d}{2}$. Step by step, we can prove that $B\left(y_{i}, d\right) \subset f\left(\mathbb{R}^{2}\right)$ for $i=2,3, \ldots$ Because $l \subset \bigcup_{i=1}^{\infty} B\left(y_{i}, d\right)$, we have $l \subset f\left(\mathbb{R}^{2}\right)$. Therefore $\mathbb{R}^{2} \subset f$ $\left(\mathbb{R}^{2}\right)$.

Next we get a theorem.

Theorem 3 Let $f: \mathbb{R}^{2} \rightarrow \mathbb{R}^{2}, f \in C^{\infty}\left(\mathbb{R}^{2}, \mathbb{R}^{2}\right)$. Let $\operatorname{det}(D f(x))>0, \forall x \in \mathbb{R}^{2}$. Suppose there exists $d>0$, so that for every $x_{0} \in \mathbb{R}^{2}$, there exist $a_{x_{0}} \in \mathbb{R}^{2}$ and $r_{x_{0}}>0$ so that $x_{0} \in B\left(a_{x_{0}}, r_{x_{0}}\right)$ and $f\left(\partial B\left(a_{x_{0}}, r_{x_{0}}\right)\right) \subset \mathbb{R}^{2} \backslash B\left(f\left(x_{0}\right), d\right)$. Let $\widetilde{D}=B\left(f\left(x_{0}\right)\right.$, d). Then every connected component of $f^{-1}(\widetilde{D})$ is bounded.

Proof. Fix $\widetilde{D}$. If $x_{0}$ satisfies the condition that $f\left(x_{0}\right)$ is the center of the disk $\widetilde{D}$, we define $C_{x_{0}}=$ the connected component of $f^{-1}(\widetilde{D})$ containing $x_{0}$. Then we have $C_{x_{0}} \subset B\left(a_{x_{0}}, r_{x_{0}}\right)$. Now we prove it. If otherwise, there exists a point in $C_{x_{0}}$ which is outside of $B\left(a_{x_{0}}, r_{x_{0}}\right)$. Note that $x_{0} \in C_{x_{0}}$ and $x_{0}$ is inside of $B\left(a_{x_{0}}, r_{x_{0}}\right)$. Also because $C_{x_{0}}$ is connected, there must be a point $p \in C_{x_{0}}$ which is on the boundary $\partial B\left(a_{x_{0}}, r_{x_{0}}\right)$. So $f(p) \in \mathbb{R}^{2} \backslash \widetilde{D}$. This contradicts the fact $p \in C_{x_{0}}$ $\subset f^{-1}(\widetilde{D})$. So we have proven that $C_{x_{0}} \subset B\left(a_{x_{0}}, r_{x_{0}}\right)$.

Next we show that the connected components of $f^{-1}(\widetilde{D})$ are all bounded. Let $X$ be any connected component of $f^{-1}$ $(\widetilde{D})$, then $X \subset f^{-1}(\widetilde{D})$. So $\exists z_{0} \in X$ so that $f\left(z_{0}\right) \in \widetilde{D}$. By our assumption, there exist $a_{z_{0}}$ and $r_{z_{0}}$ so that $z_{0} \in B\left(a_{z_{0}}, r_{z_{0}}\right)$ and

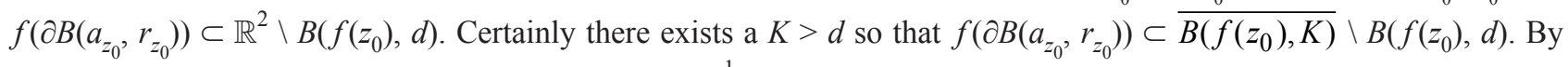
Theorem 1, for any path connected component $H$ of $f^{-1}\left(B\left(f\left(z_{0}\right), d\right)\right) \cap B\left(a_{z_{0}}, r_{z_{0}}\right)$,

$$
\left.f\right|_{H}: H \rightarrow B\left(f\left(z_{0}\right), d\right)
$$

is an onto diffeomorphism.

Let $H_{z_{0}}$ denote the path connected component of $f^{-1}\left(B\left(f\left(z_{0}\right), d\right)\right) \cap B\left(a_{z_{0}}, r_{z_{0}}\right)$ containing $z_{0}$. Then

$$
\left.f\right|_{H_{z_{0}}}: H_{z_{0}} \rightarrow B\left(f\left(z_{0}\right), d\right)
$$

is an onto diffeomorphism. We need to use this fact later in the proof.

Let $A$ denote the point $f\left(x_{0}\right) \in \mathbb{R}^{2}$. Let $B$ denote the point $f\left(z_{0}\right) \in \mathbb{R}^{2}$. Then the length of the line segment $\overline{A B}<d$ 
for $f\left(z_{0}\right) \in \widetilde{D}$. So $\overline{A B} \subset B\left(f\left(z_{0}\right), d\right)$ and $\overline{A B} \subset B\left(f\left(x_{0}\right), d\right)=\widetilde{D}$. Notice that $\left(\left.f\right|_{H_{z_{0}}}\right)^{-1}(\overline{A B})$ is defined and is a connected curve in $H_{z_{0}}$. Furthermore note that $f\left[\left(\left.f\right|_{H_{z_{0}}}\right)^{-1}(\overline{A B})\right]=\overline{A B} \subset B\left(f\left(x_{0}\right), d\right)=\widetilde{D}$. So $\left(\left.f\right|_{H_{z_{0}}}\right)^{-1}(\overline{A B}) \subset f^{-1}(\widetilde{D})$. In addition, we know $\left(\left.f\right|_{H_{z_{0}}}\right)^{-1}(\overline{A B})$ is connected. So $\left(\left.f\right|_{H_{z_{0}}}\right)^{-1}(A)$ and $\left(\left.f\right|_{H_{z_{0}}}\right)^{-1}(B)$ are in the same connected set $\left(\left.f\right|_{H_{z_{0}}}\right)^{-1}(\overline{A B}) \subset f$ ${ }^{-1}(\widetilde{D})$. Therefore $\left(\left.f\right|_{H_{z_{0}}}\right)^{-1}(A)$ is in the same connected component of $f^{-1}(\widetilde{D})$ with $\left(\left.f\right|_{H_{z_{0}}}\right)^{-1}(B)$. And we know that $(f$ $\left.\left.\right|_{H_{z_{0}}}\right)^{-1}(B)=z_{0}$, for $\left.f\right|_{H_{z_{0}}}$ is an onto diffeomorphism. So $\left(\left.f\right|_{H_{z_{0}}}\right)^{-1}(A)$ is in $X$ which is the connected component of $f^{-1}(\widetilde{D})$ containing $z_{0}$.

So for any connected component $X$ of $f^{-1}(\widetilde{D}), \exists$ a point $\tilde{x}_{0}=\left(\left.f\right|_{H_{z_{0}}}\right)^{-1}(A) \in X$ so that $f\left(\tilde{x}_{0}\right)=f\left[\left(\left.f\right|_{H_{z_{0}}}\right)^{-1}(A)\right]=A=f$ $\left(x_{0}\right)$. Therefore $\widetilde{D}=B\left(f\left(\tilde{x}_{0}\right), d\right)$, and $C_{\tilde{x}_{0}}=X$. By the first paragraph of the proof, $X=C_{\tilde{x}_{0}} \subset B\left(a_{\tilde{x}_{0}}, r_{\tilde{x}_{0}}\right.$. So $X$ is bounded.

\section{The main results and proofs}

Now we come to a theorem which is useful.

Theorem 4 Let $f: \mathbb{R}^{2} \rightarrow \mathbb{R}^{2}, f \in C^{\infty}\left(\mathbb{R}^{2}, \mathbb{R}^{2}\right)$. Let $\operatorname{det}(D f(x))>0, \forall x \in \mathbb{R}^{2}$. Suppose there exists a positive number $d>0$, so that for every $x_{0} \in \mathbb{R}^{2}$, we have $a_{x_{0}} \in \mathbb{R}^{2}$ and $r_{x_{0}}>0$ so that $x_{0} \in B\left(a_{x_{0}}, r_{x_{0}}\right)$ and $f\left(\partial B\left(a_{x_{0}}, r_{x_{0}}\right)\right) \subset \mathbb{R}^{2} \backslash B\left(f\left(x_{0}\right), d\right)$. Then $f: \mathbb{R}^{2} \rightarrow \mathbb{R}^{2}$ is an onto diffeomorphism.

Proof. First, we prove that $f: \mathbb{R}^{2} \rightarrow \mathbb{R}^{2}$ is a covering map. By Proposition $2, f: \mathbb{R}^{2} \rightarrow \mathbb{R}^{2}$ is an onto map. So $\forall y \in \mathbb{R}^{2}, \exists x_{0} \in \mathbb{R}^{2}$ so that $y=f\left(x_{0}\right)$. For $y \in \mathbb{R}^{2}$, there exists a neighborhood $V=B(y, d)=B\left(f\left(x_{0}\right)\right.$, $\left.d\right)$. Then we can decompose $f^{-1}(V)$ into the union of its connected components: $f^{-1}(V) \bigcup_{\alpha} U_{\alpha}$. Here each $U_{\alpha}$ is a connected component of $f^{-1}(V)$. And certainly $U_{\alpha} \cap U_{\beta}=\varnothing, \alpha \neq \beta$. By Theorem 3, every $U_{\alpha}$ is a bounded connected component of $f^{-1}(V)$. Then by Theorem 2, $\left.f\right|_{U_{\alpha}}: U_{\alpha} \rightarrow V$ is an onto diffeomorphism. So by the definition of covering map, $f: \mathbb{R}^{2} \rightarrow \mathbb{R}^{2}$ is a covering map. Because the base of the covering: $\mathbb{R}^{2}$ is simply connected, $f: \mathbb{R}^{2} \rightarrow \mathbb{R}^{2}$ is a homeomorphism (see [3]). And therefore $f: \mathbb{R}^{2} \rightarrow \mathbb{R}^{2}$ is an onto diffeomorphism.

A famous theorem in this field is Hadamard's global inverse function theorem. The statement of the theorem and a proof of the theorem can be found in [4] (Theorem 6.2.3 there.) As we can easily see that the properness condition of $F$ is equivalent to the condition that asks $\lim _{\|x\| \rightarrow \infty}\|F(x)\|=\infty$.

Now we can see that a direct corollary of Theorem 4 is the following version of Hadamard's global inverse function theorem in the plane.

Corollary 1 (A version of Hadamard's global inverse function theorem in the plane.) Let $f: \mathbb{R}^{2} \rightarrow \mathbb{R}^{2}, f \in C^{\infty}\left(\mathbb{R}^{2}\right.$, $\mathbb{R}^{2}$ ). Let $\operatorname{det}(D f(x))>0, \forall x \in \mathbb{R}^{2}$. Suppose $\lim _{\|x\| \rightarrow \infty}\|f(x)\|=\infty$. Then $f: \mathbb{R}^{2} \rightarrow \mathbb{R}^{2}$ is an onto diffeomorphism.

Proof. We use Theorem 4 to prove it. We check the condition of Theorem 4 for $d=1 . \forall x_{0} \in \mathbb{R}^{2}$, let $M=\left\|f\left(x_{0}\right)\right\|+$ 2. Because $\lim _{\|x\| \rightarrow \infty}\|f(x)\|=\infty$, for $M=\left\|f\left(x_{0}\right)\right\|+2>0, \exists N>0$, so that for all $x$ satisfying $\|x\|>N$, we have $\|f(x)\|>M$. So we pick $a_{x_{0}}=0, r_{x_{0}}=\max \left(2\left\|x_{0}\right\|, 2 N\right)$. We see that $x_{0} \in B\left(a_{x_{0}}, r_{x_{0}}\right)$ and $f\left(\partial B\left(a_{x_{0}}, r_{x_{0}}\right)\right)=f\left(\partial B\left(0, r_{x_{0}}\right)\right) \subset \mathbb{R}^{2} \backslash B(0, M) \subset$ $\mathbb{R}^{2} \backslash B\left(f\left(x_{0}\right), 1\right)$. So the condition of Theorem 4 is satisfied for $d=1$. Therefore $f: \mathbb{R}^{2} \rightarrow \mathbb{R}^{2}$ is an onto diffeomorphism.

Corollary 1 is one of our main results.

Definition 1 Let $f=\left(f_{1}, f_{2}\right) \in C^{\infty}\left(\mathbb{R}^{2}, \mathbb{R}^{2}\right)$, we say that $f$ increases small distances if there exists $\varepsilon>0$ such that $\| a$ $-b \|<\varepsilon$ implies $\|f(a)-f(b)\| \geq\|a-b\|, \forall a, b \in \mathbb{R}^{2}$.

Next we have

Proposition 3 Let $f=\left(f_{1}, f_{2}\right) \in C^{\infty}\left(\mathbb{R}^{2}, \mathbb{R}^{2}\right)$. Suppose $f$ increases small distances, then $\operatorname{det}(D f(x, y)) \neq 0, \forall(x, y) \in$ $\mathbb{R}^{2}$.

Proof. Let $(x, y) \in \mathbb{R}^{2}$ and $l$ be any half-line starting from $(x, y)$. Let the direction of $l$ be the direction of the vector $(\cos \xi$, $\sin \xi)$. Let the point $(x+h, y+k)$ be on $l$. (Here $h, k \in \mathbb{R}$.) Then we have 


$$
\frac{h}{\sqrt{h^{2}+k^{2}}}=\cos \xi, \frac{k}{\sqrt{h^{2}+k^{2}}}=\sin \xi
$$

By mean value theorem for functions of two variables,

$$
f_{i}(x+h, y+k)-f_{i}(x, y)=h \frac{\partial f_{i}}{\partial x}\left(x+\theta_{i} h, y+\theta_{i} k\right)+k \frac{\partial f_{i}}{\partial y}\left(x+\theta_{i} h, y+\theta_{i} k\right) .
$$

Here $\theta_{i} \in[0,1], i \in\{1,2\}$. Because $f$ increases small distances, $\exists \varepsilon>0$, so that

$$
\left(f_{1}(x+h, y+k)-f_{1}(x, y)\right)^{2}+\left(f_{2}(x+h, y+k)-f_{2}(x, y)\right)^{2} \geq h^{2}+k^{2}
$$

whenever $\sqrt{h^{2}+k^{2}}<\varepsilon$. So

$$
\begin{aligned}
& \left(\cos \xi \frac{\partial f_{1}}{\partial x}\left(x+\theta_{1} h, y+\theta_{1} k\right)+\sin \xi \frac{\partial f_{1}}{\partial y}\left(x+\theta_{1} h, y+\theta_{1} k\right)\right)^{2} \\
& +\left(\cos \xi \frac{\partial f_{2}}{\partial x}\left(x+\theta_{2} h, y+\theta_{2} k\right)+\sin \xi \frac{\partial f_{2}}{\partial y}\left(x+\theta_{2} h, y+\theta_{2} k\right)\right)^{2} \geq 1,
\end{aligned}
$$

$0 \leq \theta_{1}, \theta_{2} \leq 1$.

Let $h^{2}+k^{2} \rightarrow 0$, we get

$$
\left(\cos \xi \frac{\partial f_{1}}{\partial x}(x, y)+\sin \xi \frac{\partial f_{1}}{\partial y}(x, y)\right)^{2}+\left(\cos \xi \frac{\partial f_{2}}{\partial x}(x, y)+\sin \xi \frac{\partial f_{2}}{\partial y}(x, y)\right)^{2} \geq 1
$$

i.e.

$$
\left(\frac{\partial f_{1}}{\partial l}(x, y)\right)^{2}+\left(\frac{\partial f_{2}}{\partial l}(x, y)\right)^{2} \geq 1
$$

For all $(x, y) \in \mathbb{R}^{2}$ and all direction $l$.

Therefore we have

$$
\begin{aligned}
& \left(\frac{\partial f_{1}}{\partial x}(x, y)\right)^{2}+\left(\frac{\partial f_{2}}{\partial x}(x, y)\right)^{2} \geq 1 \\
& \left(\frac{\partial f_{1}}{\partial y}(x, y)\right)^{2}+\left(\frac{\partial f_{2}}{\partial y}(x, y)\right)^{2} \geq 1
\end{aligned}
$$

If we have 


$$
\left|\begin{array}{ll}
\frac{\partial f_{1}}{\partial x} & \frac{\partial f_{1}}{\partial y} \\
\frac{\partial f_{2}}{\partial x} & \frac{\partial f_{2}}{\partial y}
\end{array}\right|(x, y)=0
$$

for some $(x, y) \in \mathbb{R}^{2}$, it must be the case that $\frac{\partial f_{1}}{\partial x}=\alpha \frac{\partial f_{1}}{\partial y}$ and $\frac{\partial f_{2}}{\partial x}=\alpha \frac{\partial f_{2}}{\partial y}$ for some $\alpha \neq 0$. It means that for some direction $l, \frac{\partial f_{1}}{\partial l}(x, y)=0, \frac{\partial f_{2}}{\partial l}(x, y)=0$. This contradicts (1). So we have proven that

$$
\operatorname{det}(D f(x, y)) \neq 0, \forall(x, y) \in \mathbb{R}^{2} .
$$

Now we come to another main result of this paper.

Theorem 5 Let $f=\left(f_{1}, f_{2}\right) \in C^{\infty}\left(\mathbb{R}^{2}, \mathbb{R}^{2}\right)$. Suppose $f$ increases small distances, then $f: \mathbb{R}^{2} \rightarrow \mathbb{R}^{2}$ is an onto diffeomorphism.

Proof. By Proposition 3, $\operatorname{det}(D f(x)) \neq 0, \forall x \in \mathbb{R}^{2}$. Without loss of generality, suppose $\operatorname{det}(D f(x))>0, \forall x \in \mathbb{R}^{2}$. Now we check other conditions for Theorem 4. Because $f$ increases small distances, there exists $\varepsilon>0$ such that $\| a-$ $b \| \leq \varepsilon$ implies $\|f(a)-f(b)\| \geq\|a-b\|$ For any $x_{0} \in \mathbb{R}^{2}$, consider $B\left(x_{0}, \frac{\varepsilon}{2}\right)$ We see that $x_{0} \in B\left(x_{0}, \frac{\varepsilon}{2}\right)$ and $\mathrm{f}\left(\partial B\left(x_{0}, \frac{\varepsilon}{2}\right)\right) \subset$ $\mathbb{R}^{2} \backslash B\left(f\left(x_{0}\right), \frac{\varepsilon}{2}\right)$. So we choose $\frac{\varepsilon}{2}$ to be the number $d$ in Theorem 4. So the conditions in Theorem 4 is satisfied. So by Theorem $4, f: \mathbb{R}^{2} \rightarrow \mathbb{R}^{2}$ is an onto diffeomorphism.

Next we give an example for Theorem 5 .

Example 1 Let $f=\left(f_{1}, f_{2}\right)$. Here $f_{1}(x, y)=x+e^{y}, f_{2}(x, y)=x+e^{-y}, \forall(x, y) \in \mathbb{R}^{2}$. We use Theorem 5 to show that $f$ $: \mathbb{R}^{2} \rightarrow \mathbb{R}^{2}$ is an onto diffeomorphism.

Let $\left(x_{0}, y_{0}\right) \in \mathbb{R}^{2}$. We estimate the value $\left\|f(x, y)-f\left(x_{0}, y_{0}\right)\right\|^{2}$ on the circle $\left(x-x_{0}\right)^{2}+\left(y-y_{0}\right)^{2}=\lambda^{2}(0 \leq \lambda \leq 1)$.

$$
\begin{aligned}
& \left\|f(x, y)-f\left(x_{0}, y_{0}\right)\right\|^{2} \\
& =\left(f_{1}(x, y)-f_{1}\left(x_{0}, y_{0}\right)\right)^{2}+\left(f_{2}(x, y)-f_{2}\left(x_{0}, y_{0}\right)\right)^{2} \\
& =\left(x+e^{y}-\left(x_{0}+e^{y_{0}}\right)\right)^{2}+\left(x+e^{-y}-\left(x_{0}+e^{-y_{0}}\right)\right)^{2} \\
& =\left[\left(x-x_{0}\right)+\left(e^{y}-e^{y_{0}}\right)\right]^{2}+\left[\left(x-x_{0}\right)+\left(e^{-y}-e^{-y_{0}}\right)\right]^{2} \\
& =\left[\left(x-x_{0}\right)+e^{\xi}\left(y-y_{0}\right)\right]^{2}+\left[\left(x-x_{0}\right)+\left(-e^{-\eta}\right)\left(y-y_{0}\right)\right]^{2}
\end{aligned}
$$

(Here $\xi \in\left(y, y_{0}\right), \eta \in\left(y, y_{0}\right)$.)

Case(i) if $\left|x-x_{0}\right| \geq \frac{\lambda}{10}$, then 


$$
\begin{aligned}
(2) & \geq \max \left[\left(\left(x-x_{0}\right)+e^{\xi}\left(y-y_{0}\right)\right)^{2},\left(\left(x-x_{0}\right)-e^{-\eta}\left(y-y_{0}\right)\right)^{2}\right] \\
& \geq\left|x-x_{0}\right|^{2} \geq \frac{\lambda^{2}}{100}
\end{aligned}
$$

Case(ii) if $\left|x-x_{0}\right|<\frac{\lambda}{10}$, then we have

$$
\left(y-y_{0}\right)^{2}=\lambda^{2}-\left(x-x_{0}\right)^{2}>\lambda^{2}-\frac{\lambda^{2}}{100}=0.99 \lambda^{2} .
$$

So $\left|y-y_{0}\right|>\sqrt{0.99} \lambda$.

Subcase(a) if $y \geq 0, y_{0} \geq 0$, it follows

$$
\begin{gathered}
\left|\left(x-x_{0}\right)+e^{\xi}\left(y-y_{0}\right)\right| \geq e^{\xi}\left|y-y_{0}\right|-\left|x-x_{0}\right| \geq e^{0}\left|y-y_{0}\right|-\left|x-x_{0}\right| \\
\geq(\sqrt{0.99}-0.1) \lambda>0
\end{gathered}
$$

So (2) $\geq(\sqrt{0.99}-0.1)^{2} \lambda^{2}>(0.8)^{2} \lambda^{2}$.

Subcase(b) if $y \leq 0, y_{0} \leq 0$. Then

$$
\begin{aligned}
\left|\left(x-x_{0}\right)-e^{-\eta}\left(y-y_{0}\right)\right| & \geq e^{-\eta}\left|y-y_{0}\right|-\left|x-x_{0}\right| \\
& \geq e^{0}\left|y-y_{0}\right|-\left|x-x_{0}\right| \\
& \geq(\sqrt{0.99}-0.1) \lambda \\
& >0.8 \lambda>0
\end{aligned}
$$

So $(2) \geq(0.8)^{2} \lambda^{2}$.

Subcase(c) if $y y_{0} \leq 0$. Then because $\left|y-y_{0}\right| \leq \lambda, \xi \in\left(y, y_{0}\right)$, and $0 \in\left[y, y_{0}\right]$, we get $\xi \geq-\lambda$.

And

$$
\begin{aligned}
\mid\left(x-x_{0}\right)+e^{\xi}\left(y-y_{0}\right) & \geq e^{\xi}\left|y-y_{0}\right|-\left|x-x_{0}\right| \\
& \geq e^{-\lambda} \sqrt{0.99} \lambda-0.1 \lambda \\
& \geq e^{-1} \sqrt{0.99} \lambda-0.1 \lambda \\
& >\frac{1}{3}(0.9) \lambda-0.1 \lambda=0.2 \lambda>0 .
\end{aligned}
$$


So $(2) \geq(0.2)^{2} \lambda^{2}$.

Therefore in all three subcases, $(2) \geq(0.2)^{2} \lambda^{2}$.

So in both case(i) and case(ii), we have $(2) \geq 0.01 \lambda^{2}$. It follows that

$$
\left\|f(x, y)-f\left(x_{0}, y_{0}\right)\right\|^{2} \geq \frac{1}{100}\left(\left(x-x_{0}\right)^{2}+\left(y-y_{0}\right)^{2}\right)
$$

whenever $\left(x-x_{0}\right)^{2}+\left(y-y_{0}\right)^{2} \leq 1$. Now we define $F(x, y)=10 f(x, y)=\left(10 f_{1}(x, y), 10 f_{2}(x, y)\right)$. Then we see that $F$ increases small distances. By Theorem $5, F: \mathbb{R}^{2} \rightarrow \mathbb{R}^{2}$ is an onto diffeomorphism. Therefore $f: \mathbb{R}^{2} \rightarrow \mathbb{R}^{2}$ is an onto diffeomorphism.

\section{Acknowledgement}

The author would like to thank the editor(s) and the reviewer(s) for the careful reading of the manuscript and some good suggestions.

\section{References}

[1] Rabier PJ. On global diffeomorphisms of Euclidian space. Nonlinear Analysis, Theory, Methods \& Applications. 1993; 21(12): 925-947.

[2] Ding H. Diffeomorphism on path connected components and applications. Journal of Mathematical Analysis and Applications. 2020; 487(1): 123929.

[3] Lee JM. Introduction to topological manifolds. New York: Springer; 2000.

[4] Krantz SG, Parks HR. The implicit function theorem: history, theory, and applications. Boston: Birkhäuser; 2002. 